\title{
Improved quality and number of platelet count in apheresis-platelet concentrate>Buffy coat- platelet concentrate \& platelet rich plasma-platelet concentrate, assessed by study of quality parameters in II9 units of platelet concentrate
}

\begin{abstract}
Background: A successful transfusion requires whole blood to be separated into components that can be removed based on specific gravity via centrifugation. Platelet quality can then be determined using in vitro analysis of the following parameters: volume, swirling, platelet count (PLC), WBC count, and $\mathrm{pH}$. This study was performed to assess the platelet concentrate (PC) quality obtained by three different methods as per the recommended quality norms at our blood bank.

Design: Random donor platelets by platelet rich plasma-platelet concentrate (PRP-PC) Buffy coat poor-platelet concentrate (BC-PC), and single donor platelets by Apheresis-PC (APH-PC) were prepared and examined. A total of 119 units (58 BC-PC, 36 PRP-PC, and 25 APH-PC) were assessed using the following parameters: volume, swirling, PLC, and $\mathrm{pH}$.

Results: The mean volume of PRP-PC, BC-PC and APH-PC was $73.04 \pm 4.35 \mathrm{ml}$, $75.01 \pm 3.27 \mathrm{ml}$ and $272 \pm 2.98 \mathrm{ml}$ and ranged from $68-88 \mathrm{ml}, 55-82 \mathrm{ml}$ and $263-276 \mathrm{ml}$, respectively. The mean PLC of PRP-PC, BC-PC, and APH-PC were $7.95 \pm 2.31 \times 10^{10}$

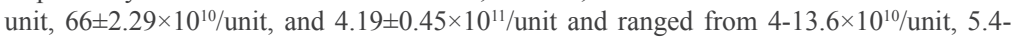
$15.4 \times 10^{10}$ unit and $3-4.7 \times 10^{11} /$ unit, respectively. The mean $\mathrm{pH}$ was $6.23 \pm 0.15$ (range: 6.0 6.8). No significant difference was observed among the three PC types. All units had a pH well above the recommended norm. PRP-PC and BC-PC units were comparable in terms of swirling, PLC per unit, and $\mathrm{pH}$ (Table 1).
\end{abstract}

Keywords: apheresis, quality parameters, platelet
Volume 5 Issue 6 - 2017

\section{Manasi PTrivedi, Mitul B Modi, Sameer B Dalsaniya, Irappa Madabhavi, Trupti Patel, Dhaval Jetly}

Department of Pathology, Gujarat Cancer \& Research Institute, Ahmedabad, India

Correspondence: Mitul B Modi, PG Resident, Department of Pathology, Gujarat Cancer \& Research Institute, Ahmedabad, India,Tel+9196620I033I, Email mitul.modi7@gmail.com

Received: March 28, 2017 | Published: December 29, 2017
Abbreviations: PC, platelet concentrate; PRP-PC, platelet rich plasma-platelet concentrate; $\mathrm{BC}-\mathrm{PC}$, buffy coat poor-platelet concentrate; APH-PC, apheresis-PC; RDP, random donor platelets; SDPs, single donor platelets; PR, percentage recovery; CCI, corrected count increment

\section{Introduction}

Since Platelets were first identified in 1981, there has been continuous and accelerating progress in our basic understanding of

Table I Parameters with quality platelet function and its utilization in various bleeding disorders. General improvement of the technique to separate platelets from whole blood and availability of plastic bags in blood banking revolutionized the field of component therapy. Platelet transfusions are the primary therapy for thrombocytopenia due to various causes. Thrombocytopenia may be due to qualitative defect, i.e. defect in platelet function or quantitative defect, i.e. decreased platelet count which can be seen in various hemato-oncological patients either due to primary disease or chemotherapy. ${ }^{1}$

\begin{tabular}{|c|c|c|c|}
\hline Quality Parameters & PRP-PC & BC-PC & A-PC \\
\hline Volume (ml) & $73.04 \pm 4.35$ & $75.01 \pm 3.27$ & $272 \pm 2.98$ \\
\hline Swirling (each day) & Present & Present & Present \\
\hline PLC (4 $4^{\text {th }}$ day) & $7.95 \pm 2.31 \times 10^{10} /$ unit & $8.66 \pm 2.29 \times 10^{10} /$ unit & 4. $19 \pm 0.45 \times 10^{11 / \text { unit }}$ \\
\hline $\mathrm{pH}\left(5^{\text {th }}\right.$ day $)$ & $6-6.4$ & $6.3-6.8$ & $6-6.6$ \\
\hline WBC contamination & $5.48 \pm 3.75 \times 10^{7} /$ unit & $4.30 \pm 3.52 \times 10^{7} /$ unit & - \\
\hline Culture & Sterile & Sterile & Sterile \\
\hline
\end{tabular}


Two types of platelet concentrates are available for transfusion; one which is the co-product of normal blood donation i.e. random donor platelets (RDP), (platelet rich plasma-platelet concentrate (PRP-PC) and buffy coat poor-platelet concentrate (BC-PC) and the other is single donor platelets (SDPs), (apheresis-PC,) collected from voluntary thrombocytapheresis donors with the help of an automated cell separator. The recommended shelf life of platelet concentrates in presently available platelet storage bags is 5 days at $22 \pm 2{ }^{\circ} \mathrm{C}$ with continuous agitation. The platelets undergo various storage changes starting from collection, processing to storage and the underlying conditions within the patients, which may affect the therapeutic benefit to the recipient.

The in vitro platelet quality can be assessed by using certain parameters (swirling, volume, platelet count and WBC count per bag and $\mathrm{pH}$ changes) and in vivo by using corrected count increment (CCI) and percentage recovery (PR) at 1 hour and 20hours post transfusion which accesses the functional platelets in circulation. In this study we have analyzed the quality of different platelet concentrates prepared by different methods as per the recommended quality norms.

\section{Material and methods}

This study was conducted in the Blood bank of G.C.R.I. Ahmedabad, a total of 119 units of platelet concentrate were selected over the period of one year (june 2014 to May 2015) for our study (Table 2). ${ }^{2}$

Table 2 No of units selected for study

\begin{tabular}{ll} 
PRP Platelets & 36 units \\
Buffy Coat PC & 58 units \\
Apheresis PC & 25 units \\
\hline
\end{tabular}

\section{Methods of preparation used}

Platelet rich plasma platelet concentrate (PRP-PC): In PRP method, PRP is separated from whole blood by 'Soft Spin' centrifugation, then platelets are concentrated by 'Hard Spin' centrifugation and the supernatant plasma is subsequently removed.

Buffy coat poor platelets concentrate (BC-PC): In Buffy coat method, whole blood is first centrifuged at 'Hard Spin' with subsequent collection of Buffy coat. Buffy coat is then centrifuged at 'Soft Spin' to concentrate platelets leaving behind the red cells and white cells.

Single donor platelet (Apheresis-PC): In Apheresis method, apheresis is carried out in a continuous flow centrifugal device, in which single donor platelet is collected and rest of the components are rein fused back to the donor (Table 3 ). ${ }^{3}$

Table 3 Methods and product obtained

\begin{tabular}{|c|c|c|c|c|c|c|c|c|c|}
\hline Method & Programme & Rpm & Rcf & Radius & Accel & Decel & Time (min) & Tem ('C) & Product obtained \\
\hline \multirow{2}{*}{\multicolumn{10}{|c|}{ BC- PC Penpol }} \\
\hline & & & & & & & & & \\
\hline & $2^{\text {nd }}$ Spin (Soft Spin) & 780 & 202 & 29.7 & 6 & 2 & 6 & 22 & Platelet Concentrate \\
\hline \multirow{2}{*}{\multicolumn{10}{|c|}{ BC-PC Fenwal }} \\
\hline & & & & & & & & & \\
\hline & $2^{\text {nd }}$ Spin (Soft Spin) & 1100 & 402 & 29.7 & 7 & 4 & 6 & 22 & Platelet Concentrate \\
\hline \multirow{3}{*}{ PRP- PC } & Ist Spin (Soft Spin) & 1800 & 1076 & 29.7 & 5 & I & 10 & 22 & RAS obtained \\
\hline & & & & & & & & & \\
\hline & $2^{\text {nd }}$ Spin (Hard Spin) & 3250 & 3507 & 29.7 & 9 & 4 & 10 & 22 & FFP, PRP obtained. \\
\hline Apheress & \multicolumn{9}{|c|}{ By Amicus Cell Separator } \\
\hline
\end{tabular}

\section{Parameters}
i. Swirling
ii. Volume of platelet concentrate
iii. Platelet count
iv. WBC count*
v. PH study
vi. Sterility

\section{Calculation}

Platelet count=platelet/cmmx1000xvolume of PC (ml)

Volume of the concentrate $=($ Wt. of the full bag-Wt. of empty bag $) /$ Sp. Gravity

WBC Count $=\mathrm{WBC} / \mathrm{cmmx} 1000 \times$ volume of PC $(\mathrm{ml})$

(Platelet and WBC count is primarily measured (/cumm) in sysmex kx30 (3 part cbc analyzer)

The swirling was evaluated by examining the units against light and scored as:

i. Score 0: Homogen turbid and is not changed with pressure.

ii. Score 1: Homogen swirling only in some part of the bag and is not clear.

iii. Score 2: Clear homogenic swirling in all part of the bag.

iv. Score 3: Very clear homogen swirling in all part of the bag.

\section{Result}

Concentrates were selected and more than $75 \%$ of each platelet Concentrate meets the criteria of quality that are comparable to the standard of WHO. The range of platelet count was $4-13.6 \times 10^{10} /$ unit

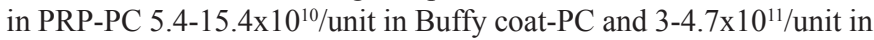
Aphaeresis-pc (Table 1, 4 \& 5). 
Table 4 Parameters scoring

\begin{tabular}{lllllllll}
\hline \multirow{2}{*}{ Scoring } & \multicolumn{2}{l}{ PRP-PC } & \multicolumn{2}{c}{ BC-PC } & \multicolumn{3}{c}{ Apheresis-PC } & \multicolumn{2}{c}{ Total } \\
\cline { 2 - 8 } & $\mathbf{n = 3 6}$ & \%age & $\mathbf{n = 5 8}$ & \%age & $\mathbf{n = 2 5}$ & \%age & $\mathbf{n = 1 1 9}$ & \%age \\
\hline 5 & 18 & 50 & 19 & 32.7 & 17 & 68 & 54 & 45.4 \\
4 & 12 & 33.34 & 28 & 48.3 & 6 & 24 & 46 & 38.6 \\
3 & 5 & 13.8 & 8 & 13.8 & 2 & 8 & 15 & 12.6 \\
2 & 1 & 2.8 & 3 & 5.2 & - & - & 4 & 3.4 \\
1 & - & - & - & - & - & - & - & - \\
\hline
\end{tabular}

Table 5 Quality Indicators for platelet concentrate

\begin{tabular}{|c|c|c|c|}
\hline S No & Quality Indicator & Benchmark & Result \\
\hline I & Rejection with Donor History of drug (aspirin and other antiplatelet drugs) & $100 \%$ & $100 \%$ \\
\hline 2 & Total Collection Time & $8-10$ minutes & $100 \%$ \\
\hline 3 & Number of times the temperature not maintained during transport blood from camp & 0 & 0 \\
\hline 4 & Component separation time (from the time of collection) & Within 6 hrs & $100 \%$ \\
\hline 5 & Volume of platelet concentrate & $50-70 \mathrm{ml}$ in $75 \%$ of the units & $100 \%$ \\
\hline 6 & $\mathrm{PH}$ of platelet concentrate & $>6$ in $75 \%$ of the units & $100 \%$ \\
\hline 7 & Platelet Count in a unit(BC- PC) & $>6 * 1010 / u l$ in $75 \%$ of the units & $100 \%$ \\
\hline 8 & Platelet Count in a unit(PRP) & $>5.5^{*} 1010 / u l$ in $75 \%$ of the units & $100 \%$ \\
\hline 9 & Platelet Count in a unit(Apheresis) & $>3.5^{*} \mathrm{I} 0 \mathrm{II} / \mathrm{ul}$ in $75 \%$ of the units & $100 \%$ \\
\hline 10 & WBC Count in a unit(BC- PC) & $<5.5^{*} 106 / \mathrm{ul}$ & $100 \%$ \\
\hline II & WBC Count in a unit (Apheresis) & $<5^{*} \mid 06 / u l$ & $100 \%$ \\
\hline 12 & WBC Count in a unit(PRP- PC) & $<5.5 * 107 / \mathrm{ul}$ & \\
\hline 13 & Bacterial contamination by culture & Sterile (I \% of all units) & $100 \%$ \\
\hline
\end{tabular}

\section{Conclusion}

Evaluations of Quality indicators conclude that platelet concentrate prepared in GCRI Blood Bank meet all the criteria for quality aspects of platelets. PRP-PC and BC-PC units were comparable in terms of swirling, platelet count per unit and $\mathrm{pH}$. As expected, we found $\mathrm{WBC}$ contamination to be less in BC-PC than PRP-PC units. Variation in volume was more in PRP-PC than BC-PC units and this suggests that further standardization is required for preparation of PRP-PC. As compared to the above two platelet concentrates, all the units of apheresis-PC fulfilled the desired quality control criteria of volume. Apheresis-PC units showed better swirling and platelet count than PRP-PCs and BC-PCs. All the platelet concentrate units had $\mathrm{pH}$ well above the recommended norm. Our study suggests though that the apheresis platelets are superior to PRP-PC and BC-PC in terms of platelet counts.

Thus, in developing countries apheresis platelets, because of their high cost and more technical expertise required may be recommended only in selected patients either when PRP-PC and BC-PC in adequate doses are not available in the inventory, or when HLA-matched platelet transfusions are indicated. Buffy coat Method is better than PRP method if we include the cost factor.

\section{Acknowledgements}

None.

\section{Conflict of interest}

The author declares no Conflict of interest.

\section{References}

1. Saran RK. Transfusion medicine technical manual. 2nd ed. India: Ministry of Health and Family Welfare; 2003. p. 341-360.

2. Holme S. Storage and quality assessment of platelets. Vox Sang 1998;74(Suppl 2):207-216.

3. Singh RP, Marwaha N, Malhotra P, et al. Quality assessment of platelet concentrates prepared by platelet rich plasma-platelet concentrate, buffy coat poor-platelet concentrate (BC-PC) and apheresis-PC methods. Asian J Transfus Sci. 2009;3(2):86-94. 